\title{
Novel Low-Loss Bandgaps in All-Silica Bragg Fibers
}

\author{
Kristopher J. Rowland, Shahraam Afshar V., and Tanya M. Monro
}

\begin{abstract}
We demonstrate that higher order bandgaps in allsilica Bragg fibers can have modes with four orders of magnitude lower confinement loss than those using the fundamental bandgap. A scheme for exploiting the higher order gaps for any specific wavelength via a global scaling of the fiber geometry is proposed. This approach provides lower losses than by reducing the confinement loss of the fundamental gap by scaling the core. Using a variety of modeling techniques, we have examined the band structure and guidance of idealized air-core all-silica Bragg fibers. It is demonstrated that the higher order, low loss, bandgaps analyzed here are uniquely accessible to single-material Bragg fibers, and are fundamentally different from the higher order gaps typically associated with depressed-index Bragg fibers such as the "Omniguide" fibers. Further analysis suggests that some of the key features of the guided modes of Bragg fibers can be understood by considering the properties of single hollow-core homogeneous dielectric waveguides ("boreholes").
\end{abstract}

Index Terms-Bragg fiber, microstructured optical fiber, optical fiber design, photonic bandgap fiber.

\section{INTRODUCTION}

$\mathbf{O}$ PTICAL fibers that can confine light within an air core have excited significant interest in recent years. These fibers are of particular promise for guiding high intensity light without damage or undesired nonlinear effects, and are also attractive for a range of applications including sensing, telecommunications, and medicine. Most of the work in this field to date has focused on silica fibers with holes arranged on a hexagonal lattice, with an air core formed by either 9 or 17 missing air holes [1].

An alternate form of air-guiding photonic bandgap fiber has recently been revived: the Bragg fiber, which has a cladding consisting of concentric layers of high and low refractive index materials [2]. Indeed, the Bragg fiber appears to have been the first proposed photonic bandgap fiber, circa 1978 [2], but its development was restricted due to fabrication technology constraints such as the requirements for material compatibility, large refractive index contrasts, and the large number of alternating layers needed to achieve reasonable confinement loss (CL). Although there have since been advances in this area [3], [4] these fabrication constraints still exist.

Recently, Vienne et al. [5] demonstrated an hollow-core single-material Bragg fiber made solely from silica and air.

Manuscript received June 30, 2007; revised September 23, 2007. This work was supported by DSTO, Australia within the Centre of Expertise in Photonics and supported in part by BAE Systems Australia.

The authors are with the Centre of Expertise in Photonics, University of Adelaide, SA 5005 Australia (e-mail: kristopher.rowland@adelaide.edu.au; shahraam.afshar@adelaide.edu.au; tanya.monro@ adelaide.edu.au).

Color versions of one or more of the figures in this paper are available online at http://ieeexplore.ieee.org.

Digital Object Identifier 10.1109/JLT.2007.911907
They showed that by using only three rings of silica-air layers connected by thin struts, such fibers can produce substantially better light confinement than what can be achieved in conventional Bragg fibers [5]. Their so-called 'OD90' fiber design is capable of an almost octave frequency range of hollow-core guidance (impossible via conventional designs) with a nominal loss of $\sim 1 \mathrm{~dB} / \mathrm{m}$ using just three pairs of cladding layers.

The modeling of an idealized (concentric annular rings with no connecting struts) version of the OD90 fiber of [5], via the asymptotic transfer matrix method [6] predicts confinement losses about 100 times lower than the total losses measured in the fabricated analog. Uranus et al. [7] calculate the CL of a geometry close to that of the fabricated OD90 fiber, claiming values very close to the experimentally measured value. This suggests that the dominant loss mechanism is indeed confinement loss and that the reason for the discrepancy with the ideal geometry is predominantly due to the presence of the connective struts. Confinement loss limited transmission has also been observed experimentally in more conventional Bragg fibers for similar near-infrared (NIR) wavelengths [4]. Recent work [8] demonstrates that the struts introduce surface modes whose CL introduces sharp features in the OD90 fiber's transmission spectrum.

Our work here focuses on the analysis of an idealized singlematerial silica Bragg fiber, where the connective struts have been ignored. The analysis of this simpler waveguide is paramount since the fundamental principles governing the behavior of the idealized case must be first understood before the effects of (potentially tunable) surface modes can be appreciated. We extend considerably upon work recently presented by the authors in [9]. In Section II, we detail the various modeling techniques used to analyze the idealized fiber and discuss points pertinent to the discussions of Section III. Section III-A analyzes the dispersion and confinement loss properties of the considered fiber, showing how there exists a second bandgap providing four orders of magnitude lower confinement loss than the fundamental. Section III-B provides insight into the guidance mechanisms responsible for this behavior. Section III-C looks at the bandgap structure of the fiber cladding in greater detail, demonstrating a nontrivial bandgap topology uniquely accessible to such Bragg fibers. Section III-D presents a way to exploit the second bandgap for potentially broadband air-core guidance with far lower confinement loss than that already observed [5].

\section{Modeling BRAGG FiberS}

Several semianalytical and numerical techniques have been used to analyze the guidance properties of Bragg fibers, including Chew's method [10], Galerkin's method [11], the transfer matrix method (TMM) [2], [11], the asymptotic method [6], [12], [13] and the finite element method (FEM) [7]. 
Galerkin's method approximates the fields by an infinite set of Laguerre-Gaussian functions, the TMM describes the fields in each layer as a linear combination of Bessel functions, and the asymptotic approach is essentially the same as the TMM except that the asymptotic forms of the Bessel functions are used. The FEM numerically solves Maxwell's equations over a mesh discretising the Bragg fiber geometry. We use the TMM here since it is the most accurate semi-analytical method [11], as confirmed by our results found with the FEM. FEM modeling can also be used to extend the analysis to real single-material Bragg fiber geometries with struts in later work.

In addition, we also analyze the behavior of the Bragg stack and borehole waveguide analogous to the Bragg fiber cladding and core respectively. As we will show later, the fundamental physics of the Bragg fiber guidance is strongly related to the optical behavior of these two structures.

\section{A. Fiber Parameters}

Since we are analyzing an idealized version of a single-material Bragg fiber (neglecting the connecting struts) we consider the conventional Bragg fiber geometry: a circular core surrounded by a cladding of two types of concentric circular layers of varying thicknesses and refractive indices. Taking $r$ as the radial coordinate, the refractive index distribution is

$$
n(r)= \begin{cases}n_{0} & \text { for } 0<r<r_{1} \\ n_{i} & \text { for } r_{i}<r<r_{i+1}\end{cases}
$$

where

$$
\begin{cases}n_{i+2}=n_{i} & \text { for } i>0 \\ t_{i+2}=t_{i} & \text { for } t_{i}=r_{i+1}-r_{i} \\ i=1,2,3, \ldots, 2 N & \end{cases}
$$

so that even or odd numbered rings have the same refractive index $\left(n_{i}\right)$ and thickness $\left(t_{i}\right)$, allowing us to define $n_{i}=\left\{n_{1}, n_{2}\right\}$ and $t_{i}=\left\{t_{1}, t_{2}\right\} \forall i=\{$ odd, even $\}$. Thus, there are $N$ pairs of layers in the cladding with width $\Lambda=t_{1}+t_{2}$ (the period). Fig. 1 is a schematic representation of an idealized single-material Bragg fiber, where $n_{2}=n_{0}=1$ (air/vacuum). This geometry has four free parameters: $R=r_{1}$ (the core radius), $t_{1}, t_{2}$, and $N$. The main case we consider here is the $N=4$ idealized single-material silica Bragg fiber considered by Vienne et al. [5], namely: $R=10 \mu \mathrm{m}, t_{1}=0.37 \mu \mathrm{m}$, $t_{2}=4.1 \mu \mathrm{m}, N=4, n_{0}=n_{2}=1$, and $n_{1}=1.45$. It is known that the $\mathrm{TE}_{01}$ mode typically produces the lowest $\mathrm{CL}$ of any mode supported by a Bragg fiber [14] and is hence the main one we consider throughout.

\section{B. Transfer Matrix Method}

We consider the wave equation

$$
\left[\nabla_{t}^{2}+\left(\frac{\omega^{2}}{c^{2}} n(r, \theta)^{2}-\beta^{2}\right)\right]\left\{\begin{array}{l}
E_{z}(r, \theta) \\
H_{z}(r, \theta)
\end{array}\right\}=0
$$

where $\nabla_{t}^{2}=\nabla^{2}-\partial^{2} / \partial z^{2}$ is the transverse Laplacian, $\omega$ is the angular frequency, and $\beta$ is the modal propagation constant. This is solved by expanding the fields using Bessell functions [2]

$$
\begin{aligned}
E_{z} & =\left[A J_{l}(k r)+B Y_{l}(k r)\right] \cos (l \theta+\phi) \\
H_{z} & =\left[C J_{l}(k r)+D Y_{l}(k r)\right] \cos (l \theta+\psi)
\end{aligned}
$$

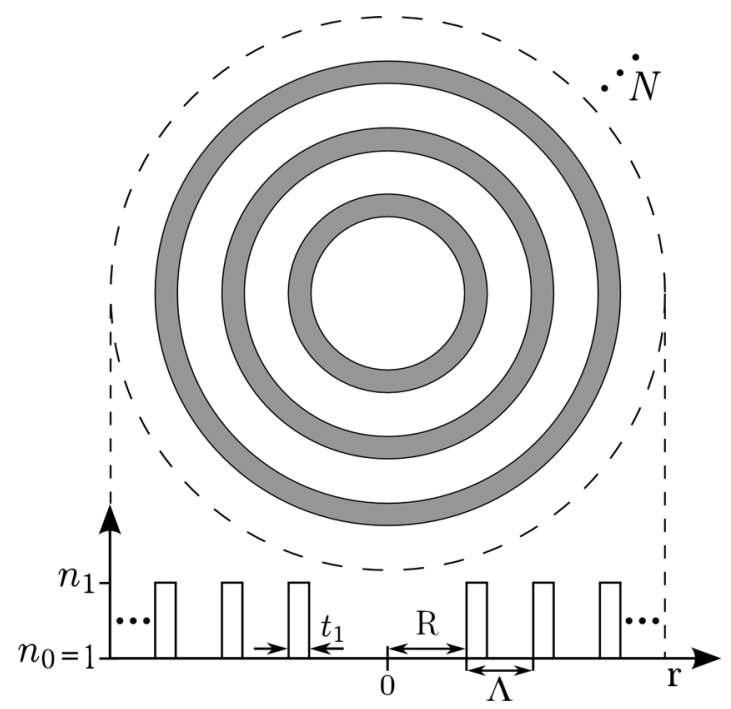

Fig. 1. Schematic representation of an arbitrary idealized single material Bragg fiber geometry with $N$ cladding pairs. The plot underneath is a slice of the profile made by any plane touching the axis of the fiber.

where $A, B, C$, and $D$ are the expansion coefficients, $\phi$ and $\psi$ are arbitrary constants, $l$ is the azimuthal quantum number (an integer) and $k=\left\{[(\omega / c) n(r, \theta)]^{2}-\beta^{2}\right\}^{1 / 2}$. The expansion coefficients between two arbitrary layers at $r=r_{i}$ are related through the dielectric waveguide boundary conditions, producing a matrix equation [2], [11]

$$
\left[\begin{array}{c}
A_{i+1} \\
B_{i+1} \\
C_{i+1} \\
D_{i+1}
\end{array}\right]=M_{i+1}^{-1}\left(r_{i}\right) M_{i}\left(r_{i}\right)\left[\begin{array}{c}
A_{i} \\
B_{i} \\
C_{i} \\
D_{i}
\end{array}\right] .
$$

Enforcing a finite field amplitude at the center and no incoming waves in the outermost layer, a complex characteristic equation can be derived from $M_{i}$ whose complex roots are the propagation constants $\beta$. This gives the effective index $n_{\text {eff }}=\beta / k_{0}$ and confinement $\operatorname{loss} \mathrm{CL}=20 \log _{10}(e) k_{0} \operatorname{Im}\left\{n_{\text {eff }}\right\}$ [15].

\section{FEM}

The FEM is implemented via the commercial FEM package Comsol Multiphysics with which we use perfectly matched layers (PMLs) [16] to calculate complex propagation constants. Since $\operatorname{Im}\{\beta\}$ is difficult to calculate accurately, a thorough convergence test as a function of the mesh and PML parameters was performed. The mesh density in the calculation domain (enclosed by the PMLs) greatly effects the value of the CL obtained and increasing the mesh density within only the $\mathrm{Si}$ rings was the best way of converging the model (a maximum element size of $0.1 \mu \mathrm{m}$ was used). Converged PML parameters must be determined first, which was done by scanning them appropriately. We found the best values for this case were: distance of inner PML edge from center $30 \mu \mathrm{m}$, PML width $2 \mu \mathrm{m}$, PML absorption coefficient [16] 1, and PML maximum mesh element size $0.15 \mu \mathrm{m}$.

We consider a quarter-plane of the fiber's cross section for all calculations, using appropriate boundary conditions to produce 


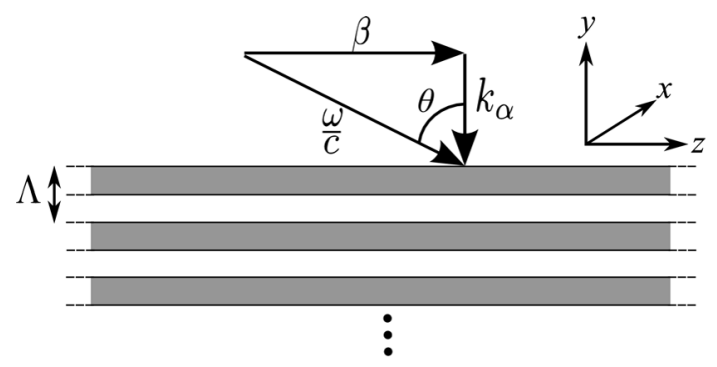

Fig. 2. Schematic representation of a Bragg stack similar to the cladding structure of the fiber in Fig. 1. Gray: $n_{1}$. White: $n_{0}=1 . N=\infty$.

TE, TM or hybrid mode classes as required. The boundary conditions of the two non-PML boundaries must be: electric conducting for TE modes, magnetic for TM modes, and a combination for the hybrid modes.

\section{Bragg Cladding Bandgaps}

In the limit of large core radius, the behavior of the cladding can be approximated by that of a planar Bragg stack [3], [4]. The work of Yeh et al. [17] derives an analytic condition determining if it is possible for light to propagate within an infinite stack. The incident plane wave's wave vector $(k)$ can be decomposed into normal $\left(k_{\alpha}\right)$ and planar components $(\beta)$, Fig. $2 . \beta$ is thus related to the propagation constant of a Bragg fiber mode made from such a cladding structure (although we strictly consider the mode's $\operatorname{Re}\{\beta\}) . k_{\alpha}$ corresponds to plane waves propagating normal to the stack (due to transmission and Fresnel reflection), where the index $\alpha$ specifies what layer type the plane wave is in $(\alpha=\{1,2\}$ for alternating layers of only two materials with thicknesses $\left.\left\{t_{1}, t_{2}\right\}\right)$. From the vector diagram in Fig. 2 it follows that $k_{\alpha}=\left\{\left[(\omega / c) n_{\alpha}\right]^{2}-\beta^{2}\right\}^{1 / 2}$, where $n_{\alpha}$ is the index of the $\alpha^{\text {th }}$ layer.

Two matrix recursion relations, one for the transverse electric (TE: electric field vector solely in the stack plane) and transverse magnetic (TM: magnetic field vector solely in the stack plane) cases, may then be constructed relating the fields of the plane waves in the stack to that of the incident wave. Up to this point, the analysis is similar to the TMM. The next step employs the Bloch-Floquet theorem, which is impossible to incorporate into a full azimuthally symmetric 2-D problem like the TMM. The theorem states that the electric field, say, must exhibit the periodicity of the stack so that $E_{K}(y, z)=E_{K}(y) e^{i K y} e^{i \beta z}$ with $E_{K}(y+\Lambda)=E_{K}(y)$ where $\Lambda$ is the stack period (Fig. 2). Plane waves in the stack are thus described as Bloch waves, reducing the recursion relation to an eigenvalue problem whose eigenvalues are the Bloch phase factors $e^{i K \Lambda}$, where $K$ is the Bloch wavenumber to be determined. The problem produces eigenvalues [17]

$$
e^{i K \Lambda}=\operatorname{Re}\{A\} \pm \sqrt{\operatorname{Re}\{A\}^{2}-1}
$$

where $A$ and $A^{*}$ are elements of the $2 \times 2$ matrix in the eigenvalue equation given by [17]

$$
A=e^{-i k_{1} t_{1}}\left[\cos \left(k_{2} t_{2}\right)-\frac{i}{2}\left(\frac{k_{2}}{k_{1}}+\frac{k_{1}}{k_{2}}\right) \sin \left(k_{2} t_{2}\right)\right]
$$

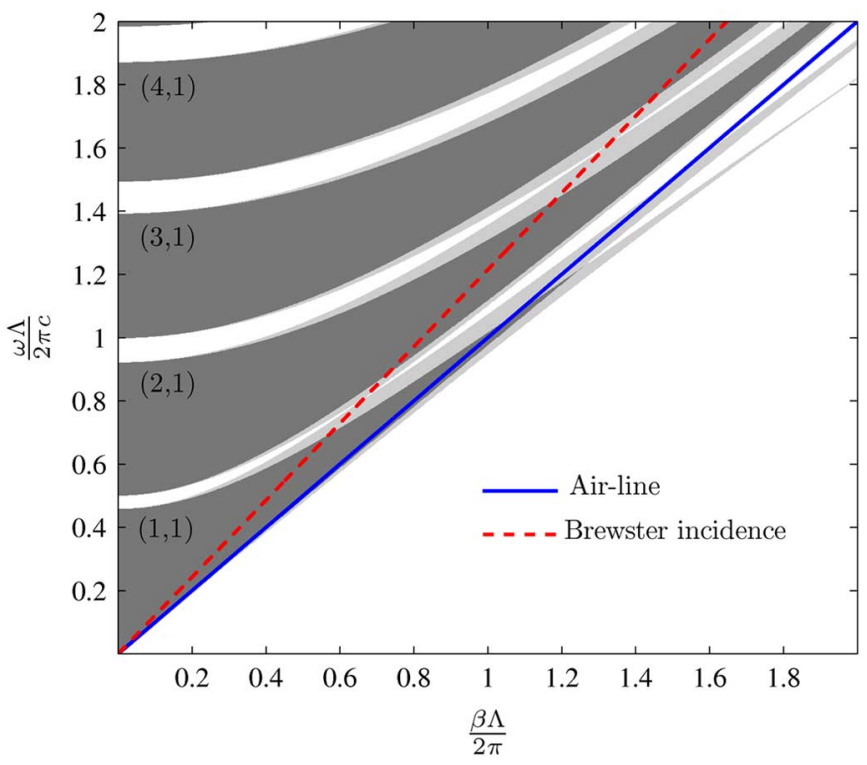

Fig. 3. Cladding bandgap map evaluated over unitless propagation constant and angular frequency. Gray regions represent the allowed bands of the Bragg stack. Above the air-line $(\beta<\omega / c)$, the TE allowed band is the dark gray region and the TM allowed band is both dark and light gray regions. The white region represents the TM bandgap and the white and light gray regions represent the TE bandgap. The TE bandgaps are labeled as defined in the text. Solid line: Air-line. Dashed line: Light-line representing Brewster angle ray incidence $\left(\theta=\theta_{B}\right)$; note that it coincides with the TM gap closure.

for TE waves and

$$
A=e^{-i k_{1} t_{1}}\left[\cos \left(k_{2} t_{2}\right)-\frac{i}{2}\left(\frac{n_{1}^{2} k_{2}}{n_{2}^{2} k_{1}}+\frac{n_{2}^{2} k_{1}}{n_{1}^{2} k_{2}}\right) \sin \left(k_{2} t_{2}\right)\right]
$$

for TM waves.

Equation (5) then gives the dispersion relation between $\omega, \beta$, and $K$ for the Bloch waves [17]

$$
K(\beta, \omega)=\frac{1}{\Lambda} \cos ^{-1}(\operatorname{Re}\{A\}) .
$$

If $K$ has an imaginary component, the Bloch wave is evanescent. Thus, only solutions satisfying the condition [17]

$$
|\operatorname{Re}\{A\}|<1
$$

correspond to waves allowed to propagate perpendicular to the Bragg stack in the $y$-direction. Its evaluation is straightforward, with $\omega$ and $\beta$ being the only free parameters. The regions in the 2-D space, $(\beta, \omega)$, satisfying (9) are allowed bands, and are shown in Fig. 3 as gray regions. Thus, the regions in between these allowed bands are the bandgaps.

From Fig. 2, $\beta=n_{0}(\omega / c) \sin \theta$ is referred to as the "lightline." In the case of $n_{0}=1$ and glancing incidence $(\theta=\pi / 2)$, we call $\beta=\omega / c$ the "air-line," Fig. 3. For fibers of sufficiently large core radius, modes of low order tend to exhibit dispersion curves $\beta(\omega)$ close to those of plane waves of glancing incidence. Decreasing the core size, or considering modes of higher order, tends to produce $\beta(\omega)$ curves corresponding to smaller $\theta$ [18].

It is well known that the Fresnel reflection efficiency increases monotonically as the incidence angle approaches $\pi / 2$, 


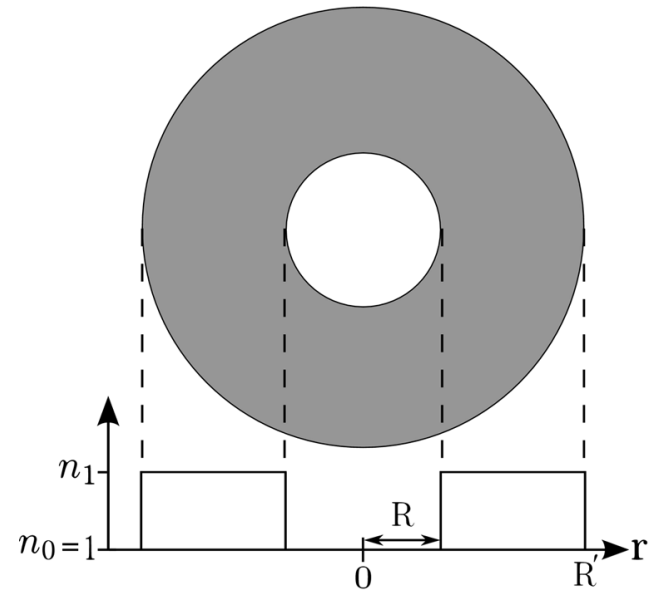

Fig. 4. Schematic of a borehole waveguide. Analysis assumes $R^{\prime} \rightarrow \infty$.

as described by the Fresnel equations [19]. Also, within a circular waveguide, such as a Bragg fiber, this glancing incidence also implies fewer 'bounces' within the core for a given axial propagation distance, hence, fewer lossy reflections from the Bragg cladding. These two points imply that a mode will typically have a lower CL when its $\beta(\omega)$ lies closer to the light-line. An explicit demonstration of this is shown below, and can also be seen in the results of Argyros et al. [18].

An important feature of the Bragg stack bandgaps is exhibited by the TM wave. Since this wave's electric field lies in the plane of incidence, it succumbs to the Brewster phenomenon [19]. In other words, if it approaches the stack at the Brewster angle $\left(\theta_{B}\right)$, it will be totally transmitted at every interface of the stack. This behavior sees the TM bandgap close up completely at all $\beta(\omega)$ lying on the light-line for $\theta=\theta_{B}$ [17], Fig. 3, and means that the TM bandgaps always lie within the TE bandgaps (for $\beta<\omega / c$ ). This behavior is often exploited when seeking to make a Bragg fiber "effectively single-moded," where substantial confinement loss discrimination between the $\mathrm{TE}_{01}$ and $\mathrm{TM}_{01}$ modes is sought so that all but the lowest loss mode are substantially attenuated for a given length of fiber [20], [21].

\section{E. Pure Hollow Core Guidance}

As will be shown later, physical insight can be drawn by considering the similarities between guidance within a Bragg fiber and a simple dielectric "borehole" (a circular air hole surrounded by a uniform dielectric, see Fig. 4). The borehole was one of the first structures considered for telecommunications [22]. Marcatili et al. [22] describe the theory of propagation within borehole structures using the general solution to the stepindex waveguide [23] where the core refractive index $\left(n_{0}\right)$ can be higher or lower than the cladding index $\left(n_{1}\right)$.

The main difference between the two cases is that the truly bound modes of the $n_{0}>n_{1}$ case are replaced by the fundamentally leaky modes of the $n_{0}<n_{1}$ case (like the borehole). In both cases, however, the general solution sees that first-order Hankel functions $\left(H_{l}^{(1)}\right)$ are used for solutions outside the core and Bessel functions of the first kind $\left(J_{l}\right)$ are used within. The dispersion relation is, thus, [23]

$$
\begin{array}{r}
{\left[\frac{J_{l}^{\prime}(u)}{u J_{l}(u)}+\frac{H_{l}^{(1)^{\prime}}(v)}{v H_{l}^{(1)}(v)}\right]\left[n_{0}^{2} \frac{J_{l}^{\prime}(u)}{u J_{l}(u)}+n_{1}^{2} \frac{H_{l}^{(1)^{\prime}}(v)}{v H_{l}^{(1)}(v)}\right]} \\
=l^{2} \frac{\beta^{2}}{k_{0}^{2}}\left(\frac{1}{u^{2}}+\frac{1}{v^{2}}\right)^{2}
\end{array}
$$

where

$$
\begin{aligned}
& u=a \sqrt{k_{0} n_{0}-\beta} \\
& v=a \sqrt{k_{0} n_{1}-\beta} .
\end{aligned}
$$

It is easily shown that (10) reduces to the typical full-vector dispersion equation for the step index fiber when we enforce $n_{0}>n_{\text {eff }}>n_{1}$, as is the case for conventional step-index fibers. It helps to note the relation between the modified Bessel function of the second kind and the first-order Hankel function: $K_{n}(z) \equiv(\pi / 2) i^{n+1} H_{n}^{(1)}(i z)$.

Considering an air core $\left(n_{0}=1\right)$ and assuming [22]

$$
\begin{aligned}
k_{0} R & \gg|l| u_{l m} \\
\left|\left(\beta / k_{0}\right)-1\right| & \ll 1
\end{aligned}
$$

the problem is simplified substantially. $R$ is the core radius and $u_{l m}$ is the $m$ th root of the equation $J_{l-1}\left(u_{l m}\right)=0$ where $l$ and $m$ are the azimuthal and radial quantum numbers of a given mode, respectively.

Inequality (13) states that the wavelength of the guided light must be much smaller than the core and that only low-order modes be considered, while (14) restricts accurate analysis to modes with $\beta$ close to the air-line. These assumptions are satisfied by the structures and modes considered here.

Using these assumptions, the dispersion relation can be manipulated to give the propagation constant analytically [22]

$$
\beta=\frac{2 \pi}{\lambda}\left\{1-\frac{1}{2}\left(\frac{u_{l m} \lambda}{2 \pi R}\right)^{2}\right\}+i\left(\frac{u_{l m}}{2 \pi}\right)^{2} \frac{\lambda^{2}}{R^{3}} \nu_{l}
$$

where

$$
\nu_{l}= \begin{cases}\frac{1}{\sqrt{n_{1}^{2}-1}} & \text { for } \mathrm{TE}_{0 m} \\ \frac{n_{1}^{2}}{\sqrt{n_{1}^{2}-1}} & \text { for } \mathrm{TM}_{0 m} \\ \frac{n_{1}^{2}+1}{2 \sqrt{n_{1}^{2}-1}} & \text { for } \mathrm{HE}_{l m} .\end{cases}
$$

An important corollary of this is that all the mode types (TE, $\mathrm{TM}$, and HE) of a given set $(l, m)$ are degenerate in $\operatorname{Re}\{\beta\}$ under this approximation. $\operatorname{Im}\{\beta\}$, however, is polarization dependent. This is a result of the Brewster phenomenon mentioned in Section II-D, and for the same reasons, sees the TM mode always having a higher loss than the TE (the hybrid modes have a loss somewhere in between since they are a combination of TE and TM modes in this regime). The arguments given above regarding modes lying close to the light-line having lowest loss are verified explicitly here as well; as $\lambda$ decreases, $\operatorname{Re}\{\beta\}$ clearly approaches $k_{0}$ monotonically while $\operatorname{Im}\{\beta\}$ is proportional to 


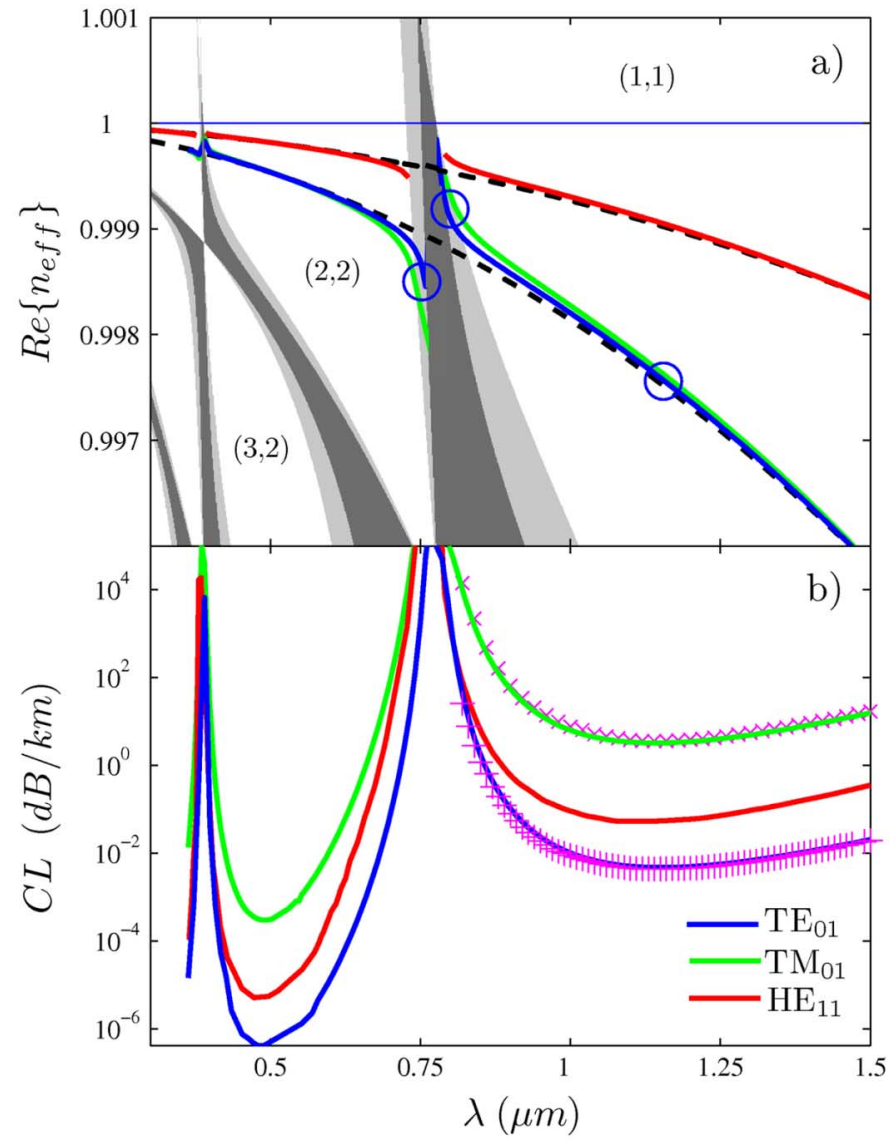

Fig. 5. Real part of the effective index (a) and the confinement loss (b) of the $\mathrm{TE}_{01}, \mathrm{TM}_{01}$, and $\mathrm{HE}_{11}$ modes found using the TMM [for $\mathrm{T}(\mathrm{E} / \mathrm{M})_{01}$ ] and FEM (for $\mathrm{HE}_{11}$ ). (a) also shows $\operatorname{Re}\left\{n_{\text {eff }}\right\}$ for the analogous borehole modes as the black dashed lines. The results are overlayed on the cladding bandgap maps, as shown in Fig. 3 but in the new coordinates $\left(\lambda, \operatorname{Re}\left\{n_{\text {eff }}\right\}\right)$. TE bandgap labels are defined in the text. Circles in (a) correspond to the points at which the fields were taken for Fig. 6. Symbols in (b) correspond to the FEM calculations of the transverse modes for the fundamental gap.

$\lambda^{2}$ and, hence, decreases. Again, as the bound rays approach glancing incidence and $\beta$ approaches the light-line, the confinement loss is reduced.

\section{RESULTS AND DISCUSSION}

\section{A. Bragg Fiber Guidance}

We have modeled the Bragg fiber using the TMM and FEM methods for the $\mathrm{TE}_{01}, \mathrm{TM}_{01}$, and $\mathrm{HE}_{11}$ modes. Dispersion curves $\operatorname{Re}\left\{n_{\text {eff }}(\lambda)\right\}$ and confinement loss CL $(\lambda)$ are shown in Fig. 5. Fig. 5(a) also shows the bandgaps associated with the cladding, as per Section II-D, demonstrating that the transition regions of the curves occur precisely at the bandgap edges.

The modes are close to the light-line and continue to approach it as the wavelength decreases, as expected from discussions above (the modes approach the behavior of a plane wave as the core becomes relatively larger). The discontinuities for each mode correspond to the transition regions of the bandgaps, with the $\mathrm{TM}_{01}$ mode exhibiting slightly higher dispersion than the

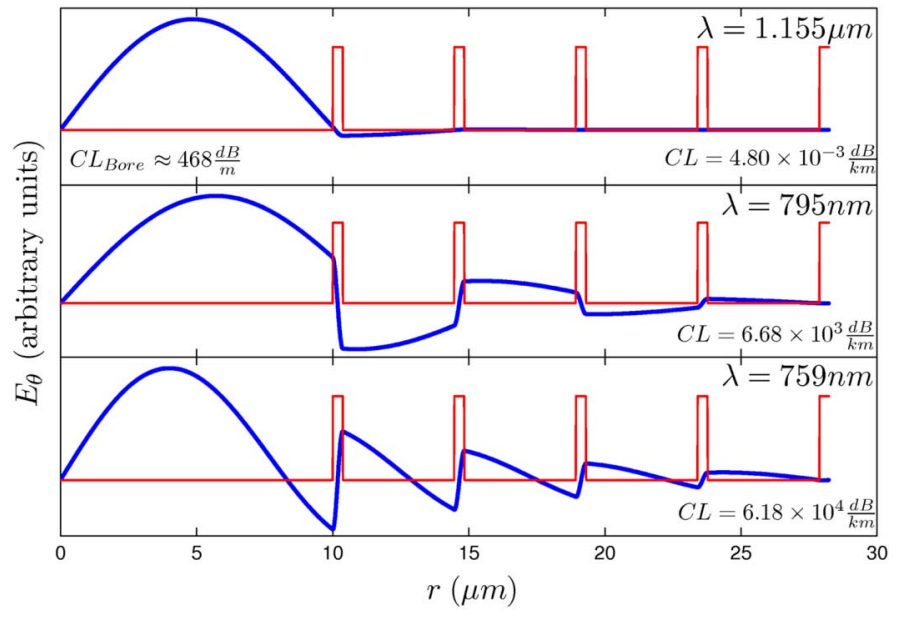

Fig. 6. Electric field of the $\mathrm{TE}_{01}$ mode at three wavelengths (depicted in Fig. 5): $1.155 \mu \mathrm{m}$, the position of $\mathrm{CL}_{\min }$ of the first gap; $795 \mu \mathrm{m}$, the low- $\lambda$ band edge of the fundamental gap; and $759 \mu \mathrm{m}$, the high- $\lambda$ band edge of the second gap. Fields are ovelayed on the refractive index profile. TE modes only have one nonzero electric field component, $E_{\theta} \hat{\theta}$. The field is azimuthally symmetric, so the field distribution has the familiar 'doughnut' shape.

$\mathrm{TE}_{01}$, as would be expected from the Brewster phenomenon arguments in Section II-D (the TM gap is always smaller than the TE gap).

Fig. 5(b) shows how the minimum $\mathrm{CL}\left(\mathrm{CL}_{\min }\right)$ reached within each bandgap is reduced as the dispersion curves approach the light line. This is expected from the discussions of Sections II-D and E, except now the effects of the bandgap edges become apparent: CL increases as the band edges are approached since more of the mode propagates within the cladding, agreeing with [18]. This is also the reason why the dispersion curves exhibit such large discontinuities over the band edges; a larger overlap of the mode with the cladding structure unsurprisingly results in a higher dispersion, forcing $n_{\text {eff }}$ to depart rapidly from its general trajectory. To demonstrate this behavior of the fields about the band edges, Fig. 6 shows the electric field distribution $\left(E_{\theta}\right)$ of the $\mathrm{TE}_{01}$ mode at specific points along its dispersion curve (Fig. 5).

The CL of the $\mathrm{TE}_{01}$ mode in the fundamental gap has a minimum of $4.80 \times 10^{-3} \mathrm{~dB} / \mathrm{km}$ at $\lambda \approx 1155 \mathrm{~nm}$. More importantly, we find that the $\mathrm{TE}_{01}$ mode in the second gap, with a minimum of $4.21 \times 10^{-7} \mathrm{~dB} / \mathrm{km}$ at $\lambda \approx 480 \mathrm{~nm}$, has $\mathrm{CL}_{\text {min }}$ four orders of magnitude lower than that of the fundamental gap, Fig. 5(b). Note also that $\mathrm{CL}_{\min }$ of all calculated modes decreases by this order, Fig. 5(b).

The difference between the propagation constants of two modes determines the extent of coupling between them due to fiber perturbations (such as surface roughness or microbending). Since the $\operatorname{Re}\left\{n_{\mathrm{eff}}(\lambda)\right\}$ of the quasi-degenerate $\mathrm{T}(\mathrm{E} / \mathrm{M})_{01}$ modes lie very close to one another in all gaps, Fig. 5(a), it is unlikely the considered fiber would be effectively single-moded. Also, by considering the borehole equivalents of the fiber modes (explained in Section III-B), it can easily be shown that only eight modes lie within the higher order bandgap and have considerably separated $\operatorname{Re}\left\{n_{\text {eff }}\right\}$ compared to the quasi-degenerate modes; the exception being $\mathrm{HE}_{21}$ which is 
also quasi-degenerate with the transverse modes. Thus, if this intermode coupling should increase the total loss of such a fiber, it is clear that the higher order bandgap may be exploited to produce modes of overall lower CL, Fig. 5(b). In this way, low-loss (albeit multimode) propagation can be achieved. We explore this further in Section III-D.

In practice, the addition of supporting struts between the cladding layers, as in [5], would alter the CL of all bandgaps by two effects: an increase in "tunnelling" through the cladding and the introduction of surface modes. Tunnelling is less of a concern for higher order bandgaps since they lie at smaller wavelengths, hence the modes would be more confined and less affected by the addition of struts. Surface modes are more complicated, introducing a complicated structure to the CL spectrum of all bandgaps by coupling with the core modes [8]. Indeed, since the CL spectrum is determined by both the cladding bandgaps and potential strut-induced surface modes, only after the idealized case is understood can the behavior of a realistic fiber be appreciated. Further, the conclusions which may be drawn from the semianalytical treatment given here would be very difficult to deduce from a realistic model alone precisely because of the increased complexity. Thus, the main focus of this work is to analyze the idealized bandgap behaviour which may be used to provide the groundwork for analysis of more realistic geometries.

Throughout this modeling, excellent agreement between the TMM and FEM models is obtained as shown in Fig. 5(b). In comparison with the asymptotic results in [5], this clearly indicates more than one order of magnitude $(20 \times)$ lower CL. Since both the TMM and FEM methods agree so well here, we conclude that the asymptotic method is not suitable for accurate modeling of CL in single-material Bragg fibers.

\section{B. Guidance Mechanisms}

Observe the behavior of $\operatorname{Re}\left\{n_{\text {eff }}\right\}$ in Fig. 5(a): all calculated modes of the Bragg fiber lie close to the trajectory of their borehole analogs. Similar behavior has already been observed in the context of surface modes [21], so its applicability here follows naturally. This deep connection between the two waveguides is quite revealing. Indeed, as discussed in Section II-E, (15) implies that $\operatorname{Re}\left\{n_{\mathrm{eff}}\right\}$ for the borehole is independent of the cladding properties; only $\lambda$ and the core radius $R$ are important. In other words, the core itself is responsible for the dispersion properties of the borehole. Since this behavior agrees so well with the Bragg fiber, we can make a similar conclusion for it as well. With the Bragg fiber, the bandgap behavior dominates as the modes reach the band edges, Fig. 5(a). These points are closely related to the work of Issa et al. [24] in which the core, not the surrounding microstructure, was found to be the dominant guidance mechanism of a polymer bandgap fiber (although it was discovered that the disorder in the fabricated cladding essentially destroyed any measurable bandgap behavior).

It is clear that $\operatorname{Re}\left\{n_{\mathrm{eff}}\right\}$ approaches the value of the refractive index of the material in which the mode has greatest overlap (air for this case). Fig. 6 also gives the CL of the Bragg fiber and equivalent borehole, showing that (at its minimum) the Bragg cladding reduces the CL significantly. Given that the $\operatorname{Re}\left\{n_{\mathrm{eff}}\right\}$ values are close in the middle of the bandgaps, we can give a

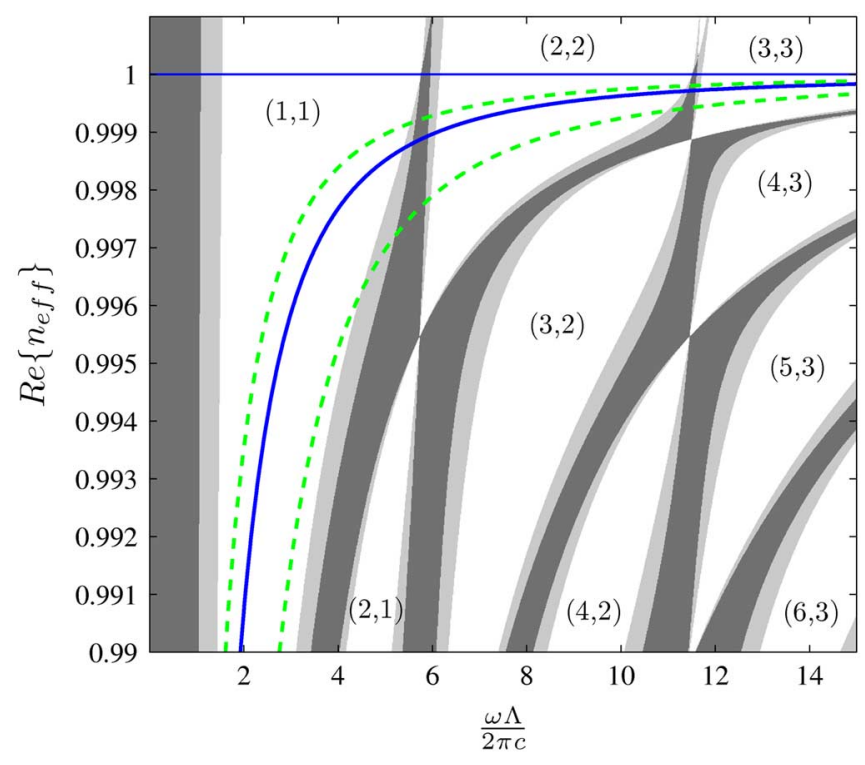

Fig. 7. The same map as Fig. 3 recast in unitless frequency and (real) effective index. The color scheme for the bandgap maps has been retained and the TE bandgaps are again labeled according to the system discussed within. Thin solid line: air-line. Solid line: The dispersion curve of the $(l, m)=(0,1)$ modes of an $R=10 \mu \mathrm{m}$ radius borehole. Dashed lines: Same as the solid line but for $R=12 \mu \mathrm{m}$ (above solid line) and $R=7 \mu \mathrm{m}$ (below solid line).

physical picture of why the CL is reduced with a Bragg cladding. Without the Bragg structure, the field in the cladding radially oscillates with exponential decay. The effect of the Bragg cladding on this tail is to maintain a similar light-material overlap (similar $\operatorname{Re}\left\{n_{\text {eff }}\right\}$ ) but reduce the overall field amplitude due to the large air-filling-fraction (Fig. 6). Thus the field amplitude at the final cladding interface will be lower than that of the borehole, reducing the $\mathrm{CL}$.

\section{Bandgap Analysis}

In order to investigate the behavior of the second bandgap further, we now consider the structure of the cladding bandgaps in more detail. Fig. 3 shows the Bragg cladding bandgap maps evaluated over unitless propagation constant and angular frequency, $(\beta \Lambda /(2 \pi), \omega \Lambda /(2 \pi c))$. This is the usual representation of Bragg stack bandgaps used in most literature.

This first representation shows where air-core modes would lie in the first bandgap since the actual dispersion curves, Fig. 5, of the low order modes are almost indistinguishable from the light-line in this representation. From this we see that this singlematerial fiber has dispersion properties quite dissimilar to other Bragg geometries where $n_{0}<\left\{n_{1}, n_{2}\right\}$. Instead of the lightline intersecting the bandgaps somewhere in the middle, here it lies close to the region where the bandgaps terminate. This is entirely due to the fact that here we have $n_{0}=n_{2}$. Clearly, the $(\beta \Lambda /(2 \pi), \omega \Lambda /(2 \pi c))$ representation makes it difficult to determine the interaction of the light-line with the bandgaps for fibers satisfying this condition.

By transforming the bandgaps to new coordinates, $\left(\omega \Lambda /(2 \pi c), \operatorname{Re}\left\{n_{\mathrm{eff}}\right\}\right)$ as in Fig. 7 , the detail close to the air-line in Fig. 3 is exposed. Immediately, we notice how the bandgap structure in the region close the air-line is very different to the gaps in Fig. 3. 
The higher order bandgaps of the Bragg stack clearly are not simply connected (Fig. 7), as may be assumed from the original representation (Fig. 3). The first-order gap is simply connected $\forall n_{\text {eff }}<1$, but all higher order gaps close at points within $n_{\text {eff }}=[0,1]$ and open up again as new gaps (this is unrelated to the TM bandgap Brewster phenomenon).

It is possible to label the TE bandgaps straightforwardly in this regime: considering the gaps coinciding with normally incident rays (a vertical line at $\beta=0$ placed on Fig. 3 ) one can label each with an index $p$, starting at 1 for the fundamental and increasing by 1 for each gap as $\omega$ increases. However, the $p$ th gap evolves into $p-1$ others as $\beta$ (or $n_{\text {eff }}$ ) increases from 0 . Thus, we may label these intermediates with another index $q \leq p$, such that any bandgap may now be referenced by $(p, q)$. The bandgaps at the extreme ends of $n_{\mathrm{eff}}=[0,1],(p, 1)$ for $n_{\mathrm{eff}}=0$ and $(p, p)$ for $n_{\text {eff }}=1$, are always open and (for $p>2$ ) the $p-2$ gaps in between are always closed. All gaps shown in Figs. 3 and 7 are labeled using this system.

Any Bragg fiber with a depressed-index core $\left(n_{0}<\right.$ $\left.\left\{n_{1}, n_{2}\right\}\right)$ is incapable of guidance within most of these higher order bandgaps. The air-line of such fibers typically intercepts the $(p, 1)$ gaps, making it impossible for any other incident ray (which must have $\theta<\pi / 2$ ) to access the higher order $q>1$ gaps. Thus, a unique property of single-material $\left(n_{0}=n_{2}\right)$ Bragg fibers is their ability to access these higher order bandgaps, particularly the $(p, p)$ gaps. This is an important point since the $(p, p)$ gaps are much wider than their lower order $(q<p)$ counterparts.

Finally, we can now identify the second gap observed in Fig. 5 as the $(p, q)=(2,2)$ gap.

It is worth noting that in practice, due to fabrication tolerances, the cladding can become aperiodic with multiple regions of uniform periodicity, such as in [5]. It can be shown that this broken periodicity combines the bandgaps associated with the different uniform regions, thus splitting them. Since the fundamental gap sits at the extremity of the long wavelength end of the bandgap spectrum, it is perturbed the least. In other words, the widths of the higher order bandgaps will decrease (in a nontrivial fashion). Because of this, it is likely that some $(p, p)$ bandgaps will produce a higher, not lower, $\mathrm{CL}_{\min }$ than those at longer wavelengths because of the relatively narrowed band edges introduced by the aperiodicity. Indeed, it can be shown that this is why the second bandgap observed by Vienne et al. [5], calculated for the idealised version of their OD90 fiber, has a higher $\mathrm{CL}_{\min }$ than the fundamental, not lower as would be expected from our treatment of purely periodic layered claddings here.

\section{Reducing Confinement Loss}

The observation that $\mathrm{CL}_{\min }$ is lower in higher order $(p, p)$ gaps than in the fundamental is a general one (for periodic claddings). This applies to any fiber with a sufficiently large $R$, such that $\operatorname{Re}\left\{n_{\text {eff }}(\lambda)\right\}$ intercepts the $(2,2)$ gap. Section III-A presents one such case. Other fiber geometries may vary in core radius $(R)$, global scale factor $(\Delta s)$, cladding layer thickness ratio $\left(t_{1} / t_{2}\right)$, or the total number of cladding ring pairs $(N)$. For all cases, higher order bandgaps will always exist and produce a lower $\mathrm{CL}_{\min }$ for sufficiently large $R$.

We now investigate the best way to reduce the confinement loss at the central wavelength of the considered Bragg fiber's
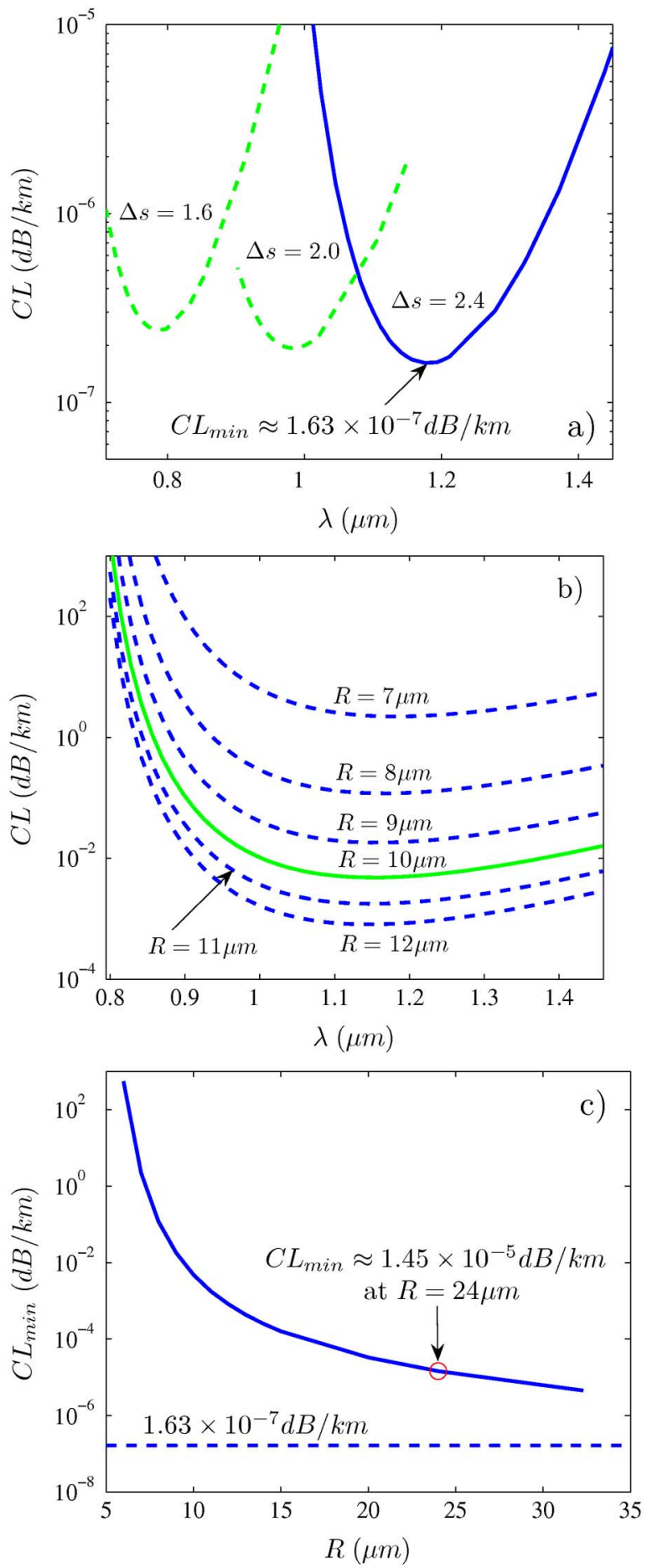

Fig. 8. The $\mathrm{TE}_{01}$ mode calculated by the TMM. (a) CL for the $(2,2)$ bandgap for global scale factors 1.6, 2.0, and 2.4. The solid curve corresponds to the scale factor for which the $(2,2)$ gap's $\mathrm{CL}_{\min }$ lies at $\lambda=1.155 \mu \mathrm{m}$ (the fundamental's $\mathrm{CL}_{\text {min }}$ position when $\Delta s=1$ ). (b) Confinement loss for the fundamental gap for various core radii $(R=7 \mu \mathrm{m} \rightarrow 12 \mu \mathrm{m})$. The original radius corresponds to the solid curve. c) $\mathrm{CL}_{\min }$ of the fundamental gap for various core radii (solid line), dashed line represents the achievable $\mathrm{CL}_{\min }$ by scaling the second gap (using $\Delta s=2.4$ ).

first gap $(\lambda \approx 1155 \mathrm{~nm})$, without altering the fiber geometry significantly (such as adding more rings to the cladding or changing the thickness ratio). As already discussed, this is tantamount to bringing $n_{\text {eff }}$ closer to the light-line. Two methods can be employed: 1$)$ Scaling the core radius $(R)$ of the fiber to reduce 
$\mathrm{CL}_{\min }$ of the fundamental gap; 2) Scaling the whole geometry up to shift the position of the $(2,2)$ gap's $\mathrm{CL}_{\min }$ to that of the fundamental $(\lambda \approx 1155 \mathrm{~nm})$. The first method works because, by increasing $R, \operatorname{Re}\left\{n_{\mathrm{eff}}\right\}$ indeed approaches 1 . This is explained via the borehole analog: (15) describes how $\beta \rightarrow k_{0}$ monotonically as $R \rightarrow \infty$. Fig. 7 explicitly shows this, with the $n_{\text {eff }}$ of the $\mathrm{T}(\mathrm{E} / \mathrm{M})_{01}$ modes for a range of $R$ overlayed on the bandgap maps. The second method is obvious from the scale invariance of the problem; since none of the fiber parameters depend on $\lambda$, by scaling $\lambda$ and the geometry by the same factor, $n_{\text {eff }}$ must be invariant, thus $\mathrm{CL}$ is also invariant up to the spatial scaling factor (since $\mathrm{CL} \propto k_{0}$ ). Also note that because of this, all calculations thus far also describe the behavior of any waveguide related via a global scale factor to the one considered (a simple but important point).

Our results in Fig. 8(a) and (b), calculated solely using the TMM, demonstrate the rate at which the position of the second gap and $\mathrm{CL}_{\min }$ of the fundamental gap change as a function of the global scale $(\Delta s)$ and core radius $(R)$, respectively. While Fig. 8(a) indicates the expected $1 / \Delta s$ relation between the minimum CL of the second gap and the global scale factor, Fig. 8(b) and (c) shows that $\mathrm{CL}_{\min }$ of the first gap exhibits an extremely rapid decrease in its rate of change for increasing $R$. This is explained by observing the position of the (equivalent borehole) dispersion curves with respect to the bandgaps as shown in Fig. 7. As $R$ decreases from $10 \mu \mathrm{m}, \operatorname{Re}\left\{n_{\text {eff }}(\lambda)\right\}$ shifts down and enters the smaller region of the $(1,1)$ gap. As the available bandgap region decreases, $\mathrm{CL}_{\min }$ is affected by both the approaching band edges and the fact that the mode is moving away from the light-line. Both these effects see that $\mathrm{CL}_{\min }$ increases rapidly as $R$ is decreased, as demonstrated in Fig. 8(b) and (c). As $R$ increases, however, $\operatorname{Re}\left\{n_{\text {eff }}(\lambda)\right\}$ approaches the light-line at a slower rate $\left[\operatorname{Re}\left\{n_{\text {eff }}\right\}-1 \propto\left(1 / R^{2}\right)\right.$ from (15)] within a region where the band edges do not change significantly, so $\mathrm{CL}_{\min }$ decreases slowly.

We now see that global scaling of the whole structure to exploit the $(p, q)=(2,2)$ bandgap is the best means of reducing $\mathrm{CL}$ at a desired wavelength, as shown in Fig. 8(c). In order to achieve the same $\mathrm{CL}_{\min }$ via increasing $R$ as by shifting the $(2,2)$ bandgap, an utterly impractical core radius would be required $(\sim 1 \mathrm{~mm})$. Exploiting the higher order bandgap in this way provides a real advantage in terms of fabrication since scaling of the fiber structure during the drawing process is much easier to implement than scaling the core radius alone. For completeness, we note that in using this method, the $(2,2)$ gap can be shifted to $1550 \mathrm{~nm}$ using a scale factor of 3.23 , giving $\mathrm{CL}_{\min } \approx$ $1.13 \times 10^{-7} \mathrm{~dB} / \mathrm{km}$.

\section{CONCLUSION}

Using a variety of modeling techniques, we have examined the band structure and guidance of idealized air-core all-silica Bragg fibers. From this we have predicted that TMM and FEM methods give more accurate CL results than the asymptotic method. By examining the bandgap structure of the fiber cladding we have determined that the Bragg stack actually exhibits a nontrivial topology of bandgaps. We have shown that single-material Bragg fibers can uniquely access higher order, wider, bandgaps unavailable to more conventional Bragg fibers (such as Omniguide fibers). A scheme for exploiting the higher order gaps at a specific wavelength via a global scaling of the fiber geometry was proposed and favorably compared to scaling only the core radius when considering the fundamental bandgap. By accessing the second-order bandgap closest to the light-line, the all-silica Bragg fiber considered can achieve a confinement loss four orders of magnitude smaller than that attainable by using the fundamental gap. By incorporating these principles into fabricable fiber designs, it is expected that the confinement loss can be reduced to levels where other loss mechanisms become dominant. We have also discussed concepts providing considerable insight into the guidance mechanisms of Bragg fibers.

\section{ACKNOWLEDGMENT}

The authors thank M. Oermann for providing the computer code used to evaluate the TMM.

\section{REFERENCES}

[1] P. S. J. Russell, "Photonic-crystal fibers," J. Lightw. Technol., vol. 24, pp. 4729-4749, 2006.

[2] P. Yeh, A. Yariv, and E. Marom, "Theory of Bragg fiber," J. Opt. Soc. Amer., vol. 68, pp. 1196-1201, 1978.

[3] B. Temelkuran, S. D. Hart, G. Benoit, J. D. Joannopoulos, and Y. Fink, "Wavelength-scalable hollow optical fibres with large photonic bandgaps," Nature, vol. 420, 2002.

[4] K. Kuriki, O. Shapira, S. D. Hart, G. Benoit, Y. Kuriki, J. F. Viens, M. Bayindir, J. D. Joannopoulos, and Y. Fink, "Hollow multilayer photonic bandgap fibers for NIR applications," Opt. Express, vol. 12, no. 8, pp. 1510-1517, 2004.

[5] G. Vienne, Y. Xu, C. Jakobsen, H. Deyerl, J. Jensen, T. Sorensen, T. Hansen, Y. Huang, M. Terrel, R. Lee, N. Mortensen, J. Broeng, H. Simonsen, A. Bjarklev, and A. Yariv, "Ultra-large bandwidth hollow-core guiding in all-silica Bragg fibers with nano-supports," Opt. Express, vol. 12, pp. 3500-3508, 2004.

[6] Y. Xu, A. Yariv, J. Fleming, and S. Lin, "Asymptotic analysis of silicon based Bragg fibers," Opt. Express, vol. 11, pp. 1039-1049, 2003.

[7] S. G. Uranus and H. Hoekstra, "Modelling of microstructured waveguides using a finite-element-based vectorial mode solver with transparent boundary conditions," Opt. Express, vol. 12, pp. 2795-2809, 2004.

[8] F. Poli, M. Foroni, D. Giovanelli, A. Cucinotta, S. Selleri, B. J. Jensen, J. Lgsgaard, A. O. Bjarklev, G. Vienne, C. Jakobsen, and J. Broeng, "Silica bridge impact on hollow-core Bragg fiber transmission properties," in Proc. OFC 2007, 2007, paper OML8.

[9] K. Rowland, S. Afshar, and T. M. Monro, "Reduction of confinement loss in all-silica Bragg bandgap fibers," in Proc. OFC 2007, 2007, postdeadline paper PDP41.

[10] W. C. Chew, Waves and Fields in Inhomogeneous Media. New York: IEEE, 1995.

[11] S. Guo, S. Albin, and R. Rogowski, "Comparative analysis of Bragg fibers," Opt. Express, vol. 12, pp. 198-207, 2004.

[12] Y. Xu, R. K. Lee, and A. Yariv, "Asymptotic analysis of dielectric coaxial fibers," J. Opt. Soc. Amer., vol. 25, no. 24, pp. 1756-1758, 2000.

[13] Y. Xu, R. K. Lee, and A. Yariv, "Asymptotic analysis of Bragg fibers," J. Opt. Soc. Amer., vol. 27, no. 12, pp. 1019-1021, 2002.

[14] S. G. Johnson, M. Ibanescu, M. Skorobogatiy, O. Weisberg, T. Engeness, M. Soljacic, S. Jacobs, J. Joannopoulos, and Y. Fink, "Lowloss asymptotically single-mode propagation in large-core OmniGuide fibers," Opt. Express, vol. 9, pp. 748-779, 2001.

[15] V. Finazzi, T. M. Monro, and D. J. Richardson, "Small-core silica holey fibers: Nonlinearity and confinement loss trade-offs," J. Opt. Soc. Amer. B, vol. 20, no. 7, pp. 1427-1436, 2003.

[16] K. Saitoh and M. Koshiba, "Full-vectorial imaginary-distance beam propagation method based on a finite element scheme: Application to photonic crystal fibers," IEEE J. Quantum Electron., vol. 38, pp. 927-933, 2002.

[17] P. Yeh, A. Yariv, and C. Hong, "Electromagnetic propagation in periodic stratified media. I. General theory," J. Opt. Soc. Amer., vol. 67, pp. 423-438, 1978. 
[18] A. Argyros, "Guided modes and loss in Bragg fibers," Opt. Express, vol. 10, no. 24, pp. 1411-1417, 2002.

[19] M. Born and E. Wolf, Principles of Optics, 7th ed. Cambridge, U.K.: Cambridge Univ. Press, 2002.

[20] I. M. Bassett and A. Argyros, "Elimination of polarization degeneracy in round waveguides," Opt. Express, vol. 10, no. 23, pp. 1342-1346, 2002.

[21] A. Argyros, I. M. Bassett, M. A. van Eijkelenborg, and M. C. J. Large, "Analysis of ring-structured Bragg fibres for single TE mode guidance," Opt. Express, vol. 12, no. 12, pp. 2688-2698, 2004.

[22] E. A. J. Marcatili and R. A. Schmeltzer, "Hollow metallic and dielectric waveguides for long-distance optical transmission and lasers," Bell Syst. Tech. J., vol. 43, pp. 1783-1809, 1964.

[23] J. A. Stratton, Electromagnetic Theory. New York: McGraw-Hill, 1941.

[24] N. A. Issa, A. Argyros, M. A. van Eijkelenborg, and J. Zagari, "Identifying hollow waveguide guidance in air-cored microstructured optical fibres," Opt. Express, vol. 11, no. 9, pp. 996-1001, 2003.

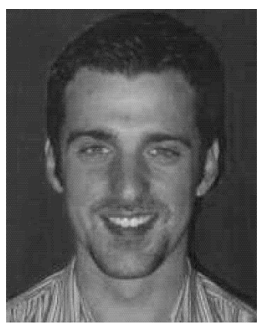

Kristopher J. Rowland received the B.Sc. degree with first class honors in 2005 from the University of Adelaide, Australia, majoring in physics and theoretical physics.

$\mathrm{He}$ is currently working toward the Ph.D. degree with the Centre of Expertise in Photonics within the School of Chemistry and Physics, University of Adelaide, Australia. His current research interests include the theory, modeling, design, and fabrication of softglass photonic bandgap fibers.

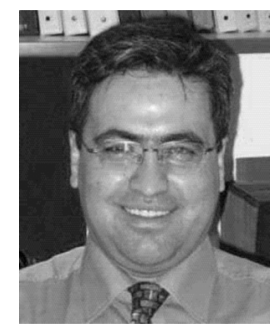

Shahraam Afshar V. received the Ph.D. degree in laser and nonlinear optics from the University of Adelaide, Australia.

He worked as a lecturer for two years with the same University. In 2001, he joined the Fiber Optics Group, University of Ottawa, Canada, as a Postdoctoral Fellow. In 2003, he received a Fellowship from the National Capital Institute of Telecommunication, Canada, to work on Brillouin scattering in photonic crystal fibers. Since 2005, he has been a Research Fellow at the Centre of Expertise in Photonics within the School of Chemistry and Physics, University of Adelaide. His current research within the Centre of Expertise in Photonics focuses on nonlinear optical phenomena in microstructured fibers, bandgap fibers, chem/biosensing using optical fibers, and teraHertz waveguides.

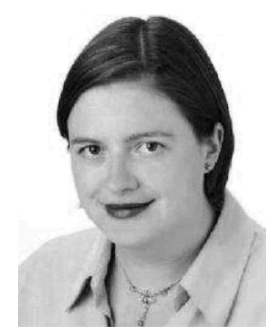

Tanya M. Monro received the Ph.D. degree from the University of Sydney, Australia, on self-written waveguides in photosensitive glasses.

From 1998 to 2004, she was with the ORC, University of Southampton, U.K., working on silica and soft-glass microstructured optical fibers. She has been the Chair of Photonics and the Director of the Centre of Expertise in Photonics within the School of Chemistry and Physics, University of Adelaide, Australia, since 2005. Her current research within the Centre of Expertise in Photonics focuses on the design, fabrication, and device applications of new classes of soft-glass microstructured optical fibers, including work on new transmission fibers, highly nonlinear fibers, chem/biosensing with new fibers, and novel fiber lasers. 